\title{
NATURAL AND ARTIFICIAL INTELLIGENCE
}

\author{
-Computor Science and Phenomenology. -
}

\section{Elmar Holenstein}

After the fascinating initial successes with computer models in psychology and neurology, there has been a sober awakening in recent years. It has become increasingly apparent that the blank spaces on the map of both sciences cannot be explored merely by developing ever more intricate versions of current computer models, but rather only by the promotion of radically different ones. This paper proposes the mode of functioning of natural languages as such an alternative model.

Natural human languages, like brains and minds, are systems endowed with the possibility of development and their actual development is under constraint of success. Successful adaptation to the tasks required of the organism by its environment is rewarded. In the following, such systems are called natural systems.

Natural languages are opposed in their mode of functioning to present-day computers in a significant respect. The mode of functioning (the functional structure) of computers is independent of the (chemical-physica1) matter (the material structure) of which they are made. Natural languages are, however, in their meaningful usages manifestly dependent upon the (usually phonic) matter of which they. are made. 
Another salient property of natural languages is intimately tied to this dependency upon matter, their plurifunctionality.

Thus the two theses set forth in this paper read as follows: (1) the mode of functioning of the brain is not independent of the particular matter of which it consists, of its particular chemical-physical structure, and (2) the structures of brain and mind are plurifunctional. Inasmuch as the plurifunctionality of a system is related to the dependence of functional structure upon material structure, these two theses are also related.

The import of the two theses - and thus of the model of natural language - is considerable for the Philosophy of Mind. In their wake, two common assumptions of recent Philosophy of Mind prove to be dogmas. They are the assumption of the underdetermination of neural or mental structures in relation to the output of the brain or mind, and the assumption of a stable functionlessness of epiphenomenal structures.

In spite of its being diametrically opposed to computer models, the model of natural language, seen from a certain perspective, is a continuation of computer models. It is an indisputable, although in recent philosophy of science little reflected fact that computers in turn owe their creation to the model of a natural system, namely the mode of functioning of human acts, concrete acts such as a goal-oriented hurling of missiles and abstract acts such as computation and other mental problem solving. 1

The simulation of specifically human acts in technical 
systems led to a startling discovery. It suddenly became pos-. sible - to the surprise of both proponents and opponents of phenomenological philosophy - to be a phenomenologist (in the sense of non-materialist, non-mechanist) without being a mentalist as we11. Computer models imply a phenomenological attitude.

The anti-materialist, anti-mechanist interpretation of computers and computer models in psychology and neurology (e.g. by G. Klaus and H. Putnam), however was not pursued with explicit recourse to phenomenological terms and theses. On the contrary, the most outspoken critics of computer models in psychology and neurology (e.g. H. Dreyfus) were the ones who recurred to phenomenological ideas. But the two theses presented here, the interrelatedness of function (sense) and matter (substance), and the plurifunctionality of structures, are also phenomenologically inspired. They are most explicit in Prague structuralism (particularly in R. Jakobson), i.e. in that branch of structural linguistics that makes frequent reference to Husser $1 .^{2}$

Since the (partial) phenomenological continuity between computer models and the model of natural languages is just as remarkable as their (partial) contrast, I shall proceed with a brief phenomenological interpretation of computer achievements. This is followed by an exemplary introduction to the two theses of the interrelatedness of function and matter and of plurifunctionality. In the last section the two dogmas of the underdetermination of neural and mental structures and the stable functionlessness of epiphenomena shall 
be subjected to critical examination.

1. The phenomenological interpretation of computer models

The historical point of departure of phenomenology was the demonstration of the autonomy of the laws of logic vis-a-vis those of psychology. This was seconded and, in the subsequent development of phenomenology, soon dominated by the thesis of the autonomy of the laws of psychology vis-a-vis those of physics. The opposing doctrines commit dire categorical mistakes. And it is precisely this view which has also become commonplace in a field deemed profane by traditional phenomenology, namely the computer sciences.

The solution of a logical problem and no less the solution of a psychological problem, e.g., the choice between two conflicting actions with regard to a system of preferences, are in principle independent of the physical constitution of the computer. The same program and thus the same problemsolving strategy can be realized in an infinity of systems with quite different physical-chemical constitutions. As Fodor puts it: "Physically different structures may obey the same psychological theory." ${ }^{3}$ Or in Putnam's more vivid formulation: "The question of the autonomy of our mental life does not hinge on and has nothing to do with that all too popular, all too old question about matter or soul-stuff. We could be made of Swiss cheese and it wouldn't matter." 4

The truly phenomenological character of this insight within the computer sciences becomes evident upon consideration of the shift in categories that accompanied it. The categories predominant in modern physics at the beginning of this 
century were thrust, pressure, attraction, repulsion, tension, load, impulse, and the like. Naturalist psychology, repudiated by phenomenology, had adopted these categories and augmented them with analogies such as reinforcement. Common to all of these categories is their implication of the notion of force. Not pioneered by any philosophers, cybernetics and information theory introduced entirely different categories into technology, categories such as goal, selection, regulation, representation, program, mode1, signal, information, and the like. In contrast to the first-named categories, the latter have nothing to do with force. Rather, they imply generic concepts such as structure and sense (i.e. goal-directedneșs).

Modern science owes its great success to the exclusion of just these humanist or - more generally - idealist categories. It has now become clear, however, that they are ontologically neutral and thus allow both a mentalist and a physicalist interpretation (i.e. realization). Even if, in the domain of the humanities, their mentalist interpretation were to prove superfluous, two aspects are still worthy of note: (1) the great advances made in this century in neurology and in biology quite generally have been possible only through the adoption of these originally humanist categories by the natural sciences, that is, through the reversal of a 300-year trend of dislodging humanist categories from more and more subject areas, and (2) there is still a categorical distinction between the first 1 ist of notions and the second, even upon aphysical interpretation of the latter. The proponents of Artificial Intelligence are justifiably charged - 
and the philosophic ones among them readily admit it - with adhering to a radical dualism when they insist that the same (functional) program can be realized by (materially) totally different physical systems. This new dualism is not, as in Descartes, a dualism between two substances, mental and physical, but rather between material structure (matter/energy) on the one hand and functional structure (function/sense) on the other, in other words a dualism between energetic and hermeneutic categories. This kind of dualism is perfectly familiar to phenomenological circles, at the latest since Ricoeur's book on Freud, ${ }^{5}$ which makes it all the more noteworthy that the anti-materialist (anti-mechanist) interpretation of computer achievements has been developed both by Marxist and analytic philosophers, but not by phenomenologists. 6

2. Two aspects of the model of natural language

An outstanding characteristic of natural languages is the fact that their mode of functioning is not independent of the matter or substance by which they are conveyed. 'Matter' and 'substance' are not to be taken metaphysical1y; they are shorthand terms for bundles of (micro-) structures which act as functional units, as vehicles of a function. In the case of language, one usually speaks of the 'sense' rather than the 'function' of an utterance. Two simple examples shall serve as illustration. In natural languages, the two semantic properties most obviously dependent upon their respective matter are connotation and 
poetic ambiguity. In many cases, they are determined by the phonic matter (structure) of their respective language.

The German words Alpdrücken and Alptraum have a literal meaning comparable to that of the English word 'nightmare,' A kobold (an elf or a mare) threatens to smother the sleeper at night. The phonic association of the 01d German word Alb ('elb') with the word Alpen ('alps') resulted in associating the feeling of being smothered with the weight of the Alps. The phonic association led not only to this interpretive shift but even to a change in spelling. The use of $a \underline{b}$ is felt to be archaic and today the word is virtually always written with a p. Both changes, semantic and graphic, could never occur in the corresponding English word 'nightmare' because of its different phonic structure.

For the sake of brevity, a pun, a piece of folk poetry, shall illustrate the dependence of poetic ambiguity on its phonic matter.

$\begin{array}{ll}\text { To be is to do. } & \text { Socrates } \\ \text { To do is to be. } & \text { Sartre } \\ \text { To be do be do. } & \text { Sinatra }\end{array}$

The phonic parallelization of these three statements effects an ambiguity of Sinatra's "To be do be do" on the one hand and of the two philosophical maxims on the other. Through the phonic similarity of Sinatra's production with the two philosophical epigrams, one is led, for a brief moment, to believe that there must also be something profound about Sinatra's line and, for a second longer moment, to suspect that possibly the sayings ascribed to the two philosophers are basically 
not much more than "to be do be do." The univocity of the three lines, read in isolation, begins to crumble.

The double meaning of the three lines is given only in English. It depends on the phonic structure (alias, the phonic matter) of each of the three verbal strings. If the pun were to be translated into the languages of the two philosophers, it would lose its point.

In a presumably older, in any case rarer version of the pun, the second sentence is attributed to Marx. The displacement of Marx by the less familiar Sartre also appears to have been phonically, i.e. materially determined and certainly not ideologically. Unlike 'Marx', the sound of 'Sartre' is related to that of 'Socrates' and 'Sinatra' (initial-S, common vowel a, similar consonantal clusters $\underline{c r-t}, \underline{r t r}, \underline{t r})$. The phonic substance in poetic contexts affects not only the meaning but even the reference! The pun is not at all an isolated case.

The second peculiarity of natural languages, their tendency to exploit pre-given structures plurifunctionally, is just as conspicuous in other natural systems whose success depends on progressive adaptation to their environment.

Swiss cheese, cited by Putnam in support of his opposing thesis of the independence of substance and function, can serve as an initial illustration. Different kinds of cheese owe their distinctive character to different micro-organisms which activate the transformation process of the milk. The bacteria that create Swiss cheese not only produce its characteristic flavor but also, by generating carbon dioxide gas, 
give rise to its characteristic holes. Other bacteria or artificial means will never be able to produce a cheese with exactly the same nutritive and aromatic content (no less and no more), not to mention the holes whıch - as everybody knows - additionally fulfill an aesthetic function for connoisseurs and a propagandistic function for dealers.

The specific cluster of functional characteristics of Swiss cheese indicates which bacteria (lactobacillus helveticus, among others) were at work for someone who enjoys eating with his stomach, mouth, and eyes. These bacteria and no others are endowed with the appropriate plurifunctional structures. The human recipient, because of his specific material structure and sensibility, is not immune to a divergent chemical structure in an artificial product, even if it were to simulate all the effects of Swiss cheese. The divergent micro-structure of the artificial product is registered if not by sight at least by by the gourmet's palate and the average consumer's stomach.

Every structural peculiarity of a food can become relevant, biologically and aesthetically, and in fact constantly does so. What applies to foodstuffs also applies to natural languages. Every structural peculiarity of a language (both grammatical and phonic, visual and auditory) can become functionally relevant, communicatively as well as aesthetically, and in fact constantly does so.

Correspondingly, artificial foodstuffs betray themselves as do artificial linguistic theories, theorjes with no psychological or neurological reality. They are either too 
simple or too powerful or both at once. They lead to deficiencies (do not achieve what they should) and/or to sideeffects (achieve more than they should). Swiss cheese can be used for many things that entirely different substances can also be used for, in principle, for example, as a computer component to simulate certain achievements of the brain. But only Swiss cheese can combine these functions, which it shares with other substances, simultaneously and exclusively with the aromatic and aesthetic effects that have made it famous. Each of its functions may be imitable in isolation, not, however, the specific cluster of its functional possibilities. Like every structure, it is equipped with a unique cluster of functional achievements.

It is not difficult to apply this point to the issue at hand. Computers, that is, models for the human brain, must be designed, whose performance depends not only on their primary functional organization, but also on their material structure, on the chemical-physical processes by which the primarily intended achievements are realized. According to the model of natural languages (and Swiss cheese), chemicalphysical processes can have a side-effect (of an expressive kind in the case of communication systems, for example), independent of the primarily intended output.

The material and functional structures of linguistic utterances are more familiar to most philosophers than the refined structure and detailed function of Swiss cheese. Therefore, a grammatical illustration shall serve to enlarge upon the thesis of the specific plurifunctionality of mate- 


\section{rial structures.}

The primary means.of indicating subject and object is word order; the first noun designates the subject, the second the object, as demonstrated by a renowned example from the Grammaire de Port-Royal: Le roi aime la reine does not have the same meaning as La reine aime le roi. The difference is indicated by the change in word order alone. Not so in Latin: Rex amat reginam. Subject and object are indicated in the Latin sentence not only by word order but by the casemarkers of the two nouns as well. Casc-marking in this sentence is an additional redundant means of indicating subject and object.

An important insight into the significance of redundancy in natural systems is revealed in this simple illustration. Reduncancies are typical not only of linguistic phenomena but of all bio-phenomena. Their function as interpreted by information theory lies in guaranteeing goal-oriented achievements. Redundancy compensates for interference. This may be its primary but it is certainly not its only function. A much more noteworthy function is that it enhances the feasibility of innovation and further cvolution.

Because of their structure, case-markers can be used not only to indicate subject and object but also, through their agreement to indicate affiliations. Magnam rex amat reginam. No Latin speaker would affiliate the adjective magnam with the adjacent noun rex, but with the noun reginam, whose case agrees with the adjective. Furthermore, the indication of subject and object through case-makers permits 
the exploitation of word order for other functions, for topicalization and emphasis.

Thus, the case-marking system, understood to have been created to guarantee through redundancy the indication of subject and object, can also - thanks to its specific structure - assume a function that departs from its primary purpose of indicating subject and object. Word order and casemarking system may be considered functionally equivalent in terms of the one task of indicating subject and object; they are not, however, omnifunctionally equivalent (equivalent in all respects). The one means can perform tasks that the other cannot because its structure is different. The structures of natural systems are characterized by the fact that, as they develop, they are exploited in an increasingly plurifunctional fashion.

This can be formulated as a general law: there are no two (material) structures that are omnifunctionally equivalent. Let us return to an extralinguistic illustration to evaluate this insight. There are several means of getting from Tokyo to Osaka - by train, car, boat, plane, bicycle, etc. We shall restrict ourselves to train and car. These two are functionally equivalent in terms of the journey to Osaka but not in terms of other services. In the shinkansen, I can read, sleep, eat during the trip. In the car, on the other hand, I can listen as I choose to the news and to music (without earphones). I can also interrupt the journey anytime or combine it with a sidetrip somewhere. Every means introduced to execute a function permits the execution of specific 
additional functions. And natural systems are quick to exploit this functional potential. Dining cars, sleepers, and reading lamps are available in trains; automobiles are equipped with radios and cassette players. The specific cluster of activities at a person a disposal on a trip from Tokyo to Osaka overdetermines his method of transportation.

A well known illustration from the recent history of linguistics of the fact that existing structures, thanks to their potential plurifunctionality, assume additional functions for which they were not (at least in the view of some linguists) originally introduced, is the semantic relevance of surface structures. A restriction of semantic information to deep structures may be an economical ideal for the theoretical linguist; a natural language system will always be quick to exploit creatively the semantic potential of surface structures, which, of ccurse, would not be denied by such a linguist either.

Unnatural speculations similar to those of mathematical linguists are also to be found in neurology. According to Dreyfus, the fact that neural pulse transmission is an all or nothing affair does not lead to the conclusion that its chemical-physical make-up implies a digital structure of the mediation of messages. A message can, in principle, be mediated by other characteristics of pulse transmission, by its frequency, for instance, and can thus be aralogically structured. This argumentation is logically correct. But it is not functional argumentation (and therefore not biological, nor phenomenological either). An evolutionary, 
adaptive system, such as the brain, can be expected, sooner or later, to exploit a condition for the mediation of messages as economical as that of the digital structure of pulse transmission - together with the analogically exploitable characteristics of pulse transmission.

The progressive evolution of a natural system is due largely to the potential for plurifunctionality that characterizes every (material) structure. Plurifunctionality is founded on the fact that there is no such thing as an entity without properties. Different entities have different properties. They can thus be plurifunctionally exploited in different ways. This also holds for redundant structures introduced primarily to safeguard the function of another structure. The redundant case-marking system with its feature of agreement permits an innovation that could never be achieved with word order.

\section{Two dogmas of recent Philosophy of Mind}

The philosophical weight of the two theses of the nonindependence of function and matter and the plurifunctionality of (material) structures is considerable. They invalidate the application of Duhem's thesis of the underdetermination of theories to linguistics and the bio-sciences (in linguistics, in the form of Quine's thesis of the indeterminacy of translation) on the one hand, and the popular defense of epiphenomenalism, on the other.

According to Duhem's thesis, the same empirical data can be explained by an infinite number of different theories. 
However, in human language, in fact in all artifacts whose constitution is steered by a code or a program, the situation is different. This is most evident in computer achievements. Input and output can be combined with each other in an infinite number of ways. The computer technologist is, however, in the fortunate position of being able to break open his black box, the computer, and ascertain code and program. The linguist's position is knottier. He must deal with two black boxes, both of which are only partially accessible: to the human mind he finds access through introspection, a frequently limited, unreliable, and correspondingly controversial means; to the brain through neurology, still in an incipient stage and correspondingly overestimated by outsiders.

The champions of the Duhem-Quine thesis maintain that even the most advanced neurology would be unable to eliminate indeterminacy. Indeterminacy applies, according to them, not only to linguistic output data, but also to the units of innate or acquired codes and programs. This is disputable, however, in view of the non-independence of matter and function and of the potential plurifunctionality of all structures. Different structures, codes and programs, categories and devices, as well as chemïcal-physical structures, are not omnifunctionally equivalent. Every structure developed to fulfill a certain function, be it in a language or in a brain, is plurifunctionally exploitable in a manner that is different from any other equivalent (with respect to this particular function) structure. 
Every structure allows the exercise (and explanation) of all those specific functions that are possible with it and that will be realized by a natural system sooner or later which has acquired this structure. The real multiplicity of output data (functions) thus becomes the index of the actual underlying structures.

Linguistic structures are overdetermined through the functions actually fulfilled by a natural language. Neurological structures are overdetermined through the functions actually fulfilled by a brain. Every theory for the explanation of particular linguistic or neural achievements is thereby not under- but overdetermined. Every alternative theory that recurs to other structures, i.e. structures with a differently exploitable plurifunctionality, inevitably proves to be not simple enough on the one hand, too powerful on the other. It is not simple enough inasmuch as it introduces several other structures to explain functions which are fulfilled by one and the same structure thanks to its specific plurifunctionality. Since these other structures display a different cluster of functions corresponding to their different features, all alternative theories prove at the same time to be too powerful. They allow additional functions, which do not occur in the system in question and, because of its specific structure, would not be possible either. All of this ultimately means that the theory of a natural system such as that of natural language or a brain, whose categories coincide with the categories of the actual code and program in the black box, is also always the simpl- 
est complete and not overly powerful theory. Duhem's discovery of the underdetermination of physical theories represented a revolution in physical theory, but it is not pertinent to the sciences of natural (biological, neurological, linguistic) systems.

The thesis of the overdetermination of the functional structure of natural systems is of course an idealization. It holds only in principle. (In this respect, however, it is no different from Duhem's and Quine's theses, which - in their fashion - also hold only in principle and are of little practical importance.) The thesis of overdetermination presupposes a system that is endowed with the possibility of evolution, the possibility of an increasingly better and progressively diversified adaptation to its enviroment, and that exploits this possibility. Complete determination is given only upon the exploitation of all possiblities. This ideal case is of purely theoretical interest. In view of the manifold factual constraints imposed upon both natural systems and scientific theory, one would be hard put to find a highly developed field of science in which alternative theories even come close to dealing as adequate1y with the same abundance of facts. In view of the principles of current scientific theory, a structure need not be exploited omnifunctiona11y, nor even multifunctional1y; plurifunctional exploitation suffices to eliminate alternative theories as less: simple and less coherent. ${ }^{7}$

The claim that a natural system with evolutional potential will inevitably exploit the plurifunctionality of its 
structures requires further elucidation. It is important to refute the dogma of the stable epiphenomenality of unavoidable side-effects in functional structures.

Epiphenomena are functionless side-effects of an organism's functionally valuable structures. It is not diffcult to discover such unavoidable side-effects of adaptive evolution. But no consideration is to be found in the literature on epiphenomenalism of the liability of functionlessness, i.e. the so-called epiphenomenality of just these sideeffects. It is claimed, for example, that the weight resulting from the polar bear's thick warm fur not only has no function, but must even be regarded as dysfunctional. This illustration smacks of scanty familiarity with biological dynamics. A natural system pressured to adapt will seek to exploit the functional potential residing in epiphenomenal and redundant structures.

Modern medicine has found that the food industry's extraction of all supposedly worthless and tastcless roughage from our food is not desirable after all since these seemingly epiphenomenal constituents have in the course of evolution long assumed manifold functions. They cultivate the growth of specific intestinal flora and guarantee a healthy, regular elimination of waste. The pressure receptors in the nerve plexus of the intestinal walls have long adjusted to a corresponding (pre-industrial) condition of fullness. It would be surprising if the weight of the polar bear's fur had not similarly affected the structure of muscles and bones, which may well be to the bear's advantage 
in more than one respect.

In addition to roughage, waste products are also considered prototypical epiphenomena. The human mind has been compared to such a product, to bile. Waste products reveal which function can be assumed by epiphenomena relatively quickly and easily. It is notably the information function. Hormones, for instance, seem to have sprung from waste products of cellular metabolism. In the course of evolution these wastes came to be used as signals. Similarly, many animals use the olfactory characteristics of their waste products for informatory purposes, just as railroad men to cite Huxley's opening illustration of epiphenomenalism use the whistle, a by-product of the excess steam escaping from the valve of an engine.

The speed with which a functionalization of epiphenomenal structures occurs must be empirically determined from system to system. Immense differences are to be found even in the information function, though it seems to be the easiest to effect. In natural languages it may arise in no time at all as demonstrated by the popular etymological exploitation of new foreign words. In other systems it may take millions of years. The eyes of our animal ancestors originally had an unconscious, non-visual, reflecting steering function. The perfection of this function resulted in a side-effect - the mirror image of the corresponding section of the environment on the eye. It took millions of years to be able to exploit this initially purely epiphenomenal image functionally, namely perceptually, as we do today. 
Not only redundant structures (which once merely functioned as a guarantee), and epiphenomenal structures (which were once functionless by-products of functional structures), but even irritating, dysfunctional by-products of functional structures, known in information theory as noise, are functionally exploited in natural systems. Noise is a typical term in the theory of artifical communications systems. Information theory denies any function to noise - just as it assigns a merely safeguarding function to redundancy. However, in natural communications noise (as technically defined) is an ephemeral phenomenon. It is as labile as epiphenomena are. In natural communications systems, noise is in fact very quick to assume an information function. Telephone, radio, and records with their background noise as well as old movies that flicker on the screen are both good illustrations. Neither noise nor flicker have to be heard or seen very long to take on an expressive function. They serve to identify the sender as old-time, for example, and thus as having a certain flair and evoking a whole range of emotions. Even the background noise, varying according to the frequency of the broadcasting station and registered by the human ear below the level of consciousness, comes to have a familiar, almost comforting effect. Music and news on a station to whose underlying sounds the ear has become attuned are allegedly given a higher rating than comparable broadcasts on other stations. A Chaplin film that neither flickers nor jerks is like an antique piece of furniture that has lost its patina in restoration. 
Language sciences have been blindly and unimaginatively oriented toward the technology of artificial communications systems far too long. 'Noise' is a central concept in the theory of technical communication systems. It is quite another story in the theory of natural languages. Language psychologists have repeatedly voiced the supposition that apparently by way of evolution - man cannot suffer any meaninglessness. He will try to make sense out of a phenomenon that appears to make no sense and, since nothing occurs in isolation but always in an associative context, he will succeed.

The arguments discussed in this paper add fresh weight to two traditional suppositions of Philosophy of Mind that have commonly been taken for granted. (1) If one thinks in terms of biological analogies, then it would seem that the function a human mind is most likely to assume is an informational one. (2) If one thinks in terms of the analogy of natural language systems, then it would seem that mind - as a redundant process vis-à-vis brain processes - is (will be) beneficial (in a way that the brain alone could not be) to the creativity of mind-endowed animals, and this due to the specific plurifunctionality of its structure. But what are the neural and mental structures underlying the specifically human cluster of functional achievements? In the long race to answer these questions, both neurology and phenomonology are still at the starting line. 


\section{Annotations}

1) The creation of the first computer models was based on the introspection of the procedure in the planning and execution of corresponding human acts. See H. Schnelle; "Introspection and the Description of Language Use," in A Festchrift for Native Speakers, ed. by F. Coulmas, The Hague: Mouton, 1981, $116 \mathrm{ff}$, and also S. Watanabe, Knowing and Guessing, New York: Wiley, 1969, 387.

2) See E. Holenstein, Roman Jakobson's Approach to Language: Phenomenological Structuralism, Bloomington, Ind.: Indiana University Press, 1976 (Jakobson: Genshōgakuteki Kozōshugi, Tokyo: Hakusuisha, 1983), and "Von der Poetik und der Plurifunktionalität der Sprache," in Roman Jakobson, Poetik, Frankfurt: Suhrkamp, 1979, 7-60.

3) H. Putnam's rendition, Philosophical Papers 2, Cambridge University Press, 1975, 392.

4) Ibid., 291 .

5) De 1'interprétation - Essai sur Freud, Paris: Seuil, 1965.

6) For the East German Marxist G. Klaus (Philosophie in kybernetischer Sicht, Berlin: Dietz, 1961), cybernetics and information theory confirm (holistic) dialectical materialism's critique of the (atomistic) mechanistic materialism.

7) See also the argumentations of H. Putnam, op.cit., 337 ff.; N. Chomsky, Language and Responsibility, New York: Pantheon, 1979, 166; Z. W. Pylyshyn, "Computation and Cognition," in The Behavioral and Brain Sciences 3, 1980, $123 \mathrm{ff}$. 